\title{
Design of a new type of variable magnetic excitation sensor
} Xinyuan Cao ${ }^{1, a}$, Xuanyi Gao 2,b ${ }^{2}$ Weimin Zhang ${ }^{1, c}$, Zhongchao Qiu ${ }^{1, d}$

${ }^{1}$ School of Mechanical Engineering, Beijing Institute of Technology, Beijing 100081, China;

${ }^{2}$ School of Information and Electronics, Beijing Institute of Technology, Beijing 100081, China;

E-mail:acaoxinyuanbj@sina.com, bgaoxuanyi@bit.edu.cn, 'czhangwmbit@163.com, d448347664@163.com

Key words:Magnetic detection; AMR; integrate; MMM; MFL; MBN

Abstract:An integrated non destructive testing system using AMR magnetic sensor is manufactured which contains detection technology of metalmagnetic memory(MMM), magnetic flux leakage(MFL) and Barkhausen(MBN)in order to detect the defects of ferromagnetic materials rapidly. The test of 45 steel specimens with prefabricated cracks in different directions and stress concentration zone was carried out. The results show that the system can detect the defects sensitively and evaluate the stress concentration quantitatively.

\section{Introduction}

Due to large defects can lead to scrap parts, residual stress will cause deformation, imperfections and residual stress are the eternal theme of detection in the state of using magnetic nondestructive sensor to detect ferromagnetic. So, is there any way to make a unified design of the two?If we can achieve a unified design, we will simplify the use of the process and facilitate the practical application of the project[1].In this paper, a new kind of sensor is presented, which can pick up different signals according to different testing requirements, and then make the best evaluation of condition of the equipment. The test of 45 steel specimens with prefabricated cracks in different directions and stress concentration zone was carried out.

\section{Analysis of the feasibility of integration}

With the development of Nondestructive Testing Technology(NDT), it is very difficult to determine the damage of the workpiece with a single detection technique.Developing non destructive testing integration technology is a necessary means to ensure the safety of equipment[2]. In essence, the MMM signal is a weak static magnetic field which can reflect the information of the defects, while the MFL signal is the active magnetic field which can reflect the position and size of the defect in the strong magnetic field, they all belong to the leakage field[3].MBN measuring the variation of internal magnetic properties of ferromagnetic specimens, which is used to measure stress.Their detection platform common to each other, just different in magnetic condition, collection frequency and so on. Therefore, the three means are similar in essential principles, and the combination of the them can complement each other to reflect defects and stress, so as to realize the nondestructive evaluation of ferromagnetic components .

\section{Sensor design}

This sensor make MMM, MFL and MBN integrated which consists of reset circuit sensorprobe,conditioning circuit, excitation circuit, excitation coil and collection card. The structureis shown in

Fig. 1. 


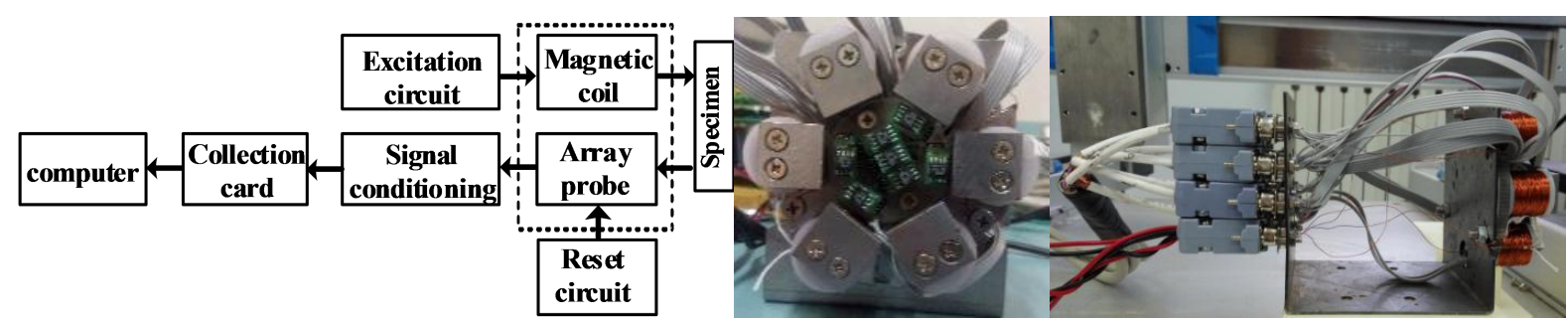

Fig .1Structure flow chart of sensor

Fig. 2Probe structure of sensor

Probe structure of sensor. Probe part is composed of 6 jaw magnetic yoke, and the signal receiving part is made up of 14 magnetic sensors, each two chips are combined vertically to form a sensing modulemeasuring the magnetic field in the direction of $X$ and Zas shown in Fig.2. Two coils in six claw yoke's relative claw connected into a group with the yoke plate, iron core, pole shoe and workpiece constitute a magnetic circuit, six claw magnetic yoke can generate three groups of magnetic circuitin space.

Circuit of sensor. Excitation circuit generate sine wave, then phase shift of the three channel sine wave is carried out by the phase shift circuit, finally, the signal amplified by amplifier circuit. Using pulse reset circuit to improve the sensitivity of the magnetic sensor[4]. The Magnetic signal is amplified by the amplified filter circuit then collected by the acquisition card and sent to the computer. circuit of sensor is shown in Fig. 3.

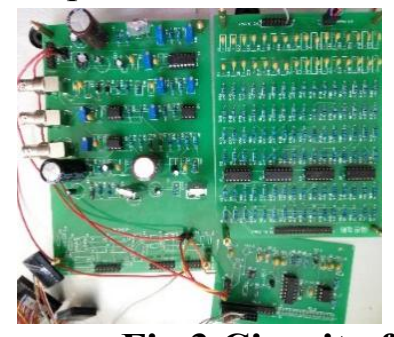

Fig.3 Circuit of sensor

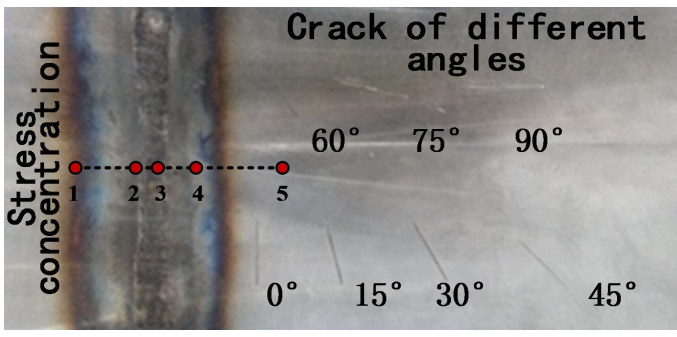

Fig.4 The specimen.

\section{Experiment and result analysis}

Preparation of specimens and analysis of residual stress in $\mathbf{X}$ - ray. The stress concentration and crack defects was made on 45 carbon steel plate specimen whose size is $400 \mathrm{~mm}($ long $) \times 200$ $\mathrm{mm}$ (broad) $\times 10 \mathrm{~mm}$ (thick), The thickness of the specimen is used in order to conform to the actual project as far as possible. Among them, the crack size is $40 \mathrm{~mm} \times 0.5 \mathrm{~mm} \times 0.5 \mathrm{~mm}$, and the angle is interval 15 degrees, The specimen is shown in Fig. 4. For the five measurement points shown in Fig. 4 , measuring the residual stress in the direction of the length of the specimen through portable $\mathrm{X}$ ray stress analyzer.

Experimental process and results.In this paper, the $\mathrm{X}$ direction magnetic field information measured by the center sensor is analyzed and illustrated. When MMM, DC MFL and AC MFL are used, the acquisition frequency is set to $1000 \mathrm{~Hz}$, when using $\mathrm{MBN}$ is $8000 \mathrm{~Hz}$, all meet the conditions of the sampling theorem[5]. Because the collected signal is susceptible to environmental factors such as noise, wavelet analysis method is used to deal with the collected signal, and chose the wavelet function db10[6].

MMM test.MMM signal is not generated by active magnetic excitation, it receives the abnormal magnetic signal of ferromagnetic specimen, the passive signal can be picked up without any excitation. MMM signal has a certain degree of reflection of stress concentration area and the defect signal, but the signal amplitude is not strong, MMM signal is not very clear so it is difficult to determine the existence of defects and stress quantitatively[7].

DC MFL test.Compared with the AC MFL signal, the DC MFL signal has a deeper magnetic effect, which is more favorable for the detection of deep defects[8]. At this time adjusting the frequency at $10 \mathrm{~Hz}$, power amplifier output voltage at $3 \mathrm{~V}$. The amplitude of the DC MFL signal is better than that of MMM signal, with more and more strong magnetic field, this phenomenon is more and more obvious[9]. But the unidirectional DC MFL test is bad for the detection of crack in different directions, when crack $75^{\circ}$ is detected, the amplitude is very small as shown in Fig. $\mathbf{5}$. 


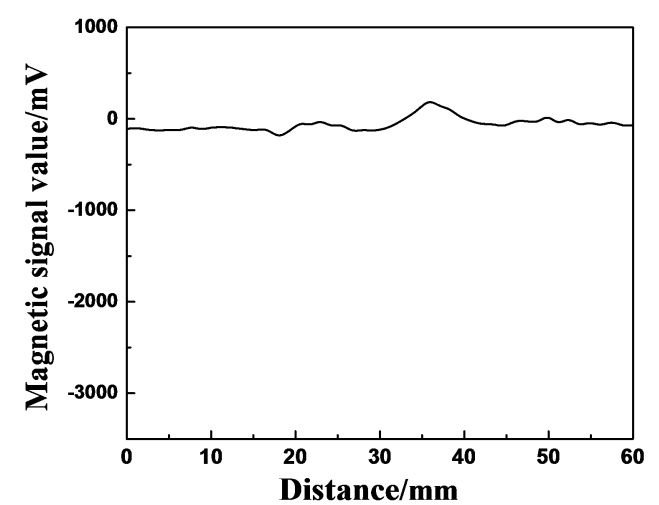

Fig. 5 DC MFL signal for crack $75^{\circ}$

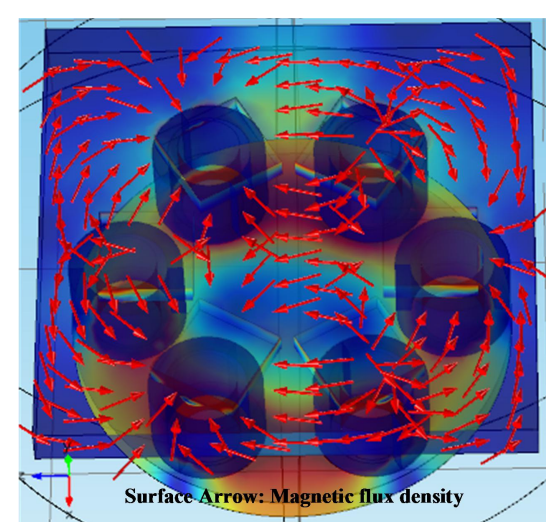

Fig. 6 Sensor magnetic field simulation diagram

AC MFL test. AC MFL signal is easier to produce, and can be formed by the sinusoidal signals with different phases, its basic principles are asshown in Eq. 1: Sinusoidal excitation current vector signal with the same size, frequency and phase difference of $120^{\circ}$ are respectively communicated in the adjacent magnetic yoke coil and the magnetization vector field with uniform change in direction is synthesized.

$$
\left\{\begin{array}{c}
I_{x}=I_{0} \sin \left(\omega t+\phi_{0}\right) \\
I_{y}=I_{0} \sin \left(120^{\circ}+\omega t+\phi_{0}\right) \\
I_{z}=I_{0} \sin \left(-120^{\circ}+\omega t+\phi_{0}\right)
\end{array}\right.
$$

In the equation, $\omega=2 \pi f, f$ is excitation current frequency, $\phi_{0}$ is Initial phase, $I_{0}$ is amplitude, by the Maxwells equations knowable, the inducted magnetic field in the test piece is basically constant in size and rotate in direction with time. Fig.6 is the results of simulation at a certain time for the magnetic field of the sensor by using of COMSOL. Under the condition of AC MFL, the electromagnetic field vector is deflected, which makes the sensor sensitive to cracks in different directions. Fig.7 is AC MFL signal for crack90 ${ }^{\circ}$.

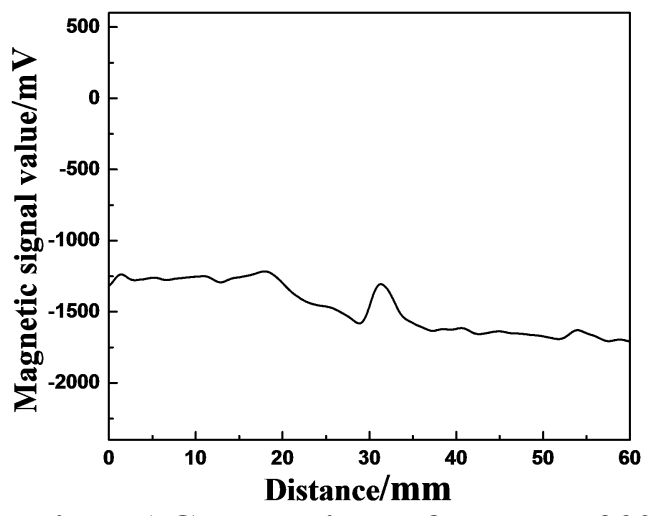

Fig. 7 AC MFL signal for crack $90^{\circ}$

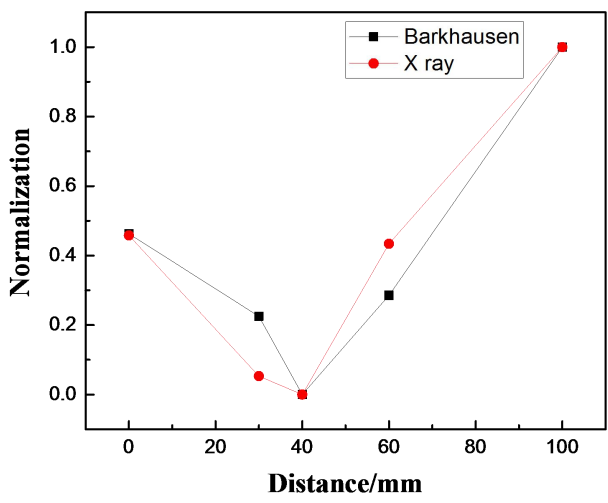

Fig. 9 Barkhausen noise mean square value compared with $X$ ray diffraction

MBN test. Numerous technical documents have confirmed that MBN signal and residual stress have good correlation. In fact, the main difference between MBN signal and the MFL signal is power and signal acquisition frequency. Adjusting the excitation frequency at $30 \mathrm{~Hz}$ and the power amplifier output voltage at $1 \mathrm{~V}$ during experiment. Calculating the mean square value of MBN signal of 5 measurement points in figure 4, detecting five times per point then taking the average, the results are shown in Table 1[10]. 
Table1The mean square value $\left(\mathrm{mV}^{2}\right)$ of arkhausen noise

\begin{tabular}{ccccccc}
\hline Testpoint & First & Second & Third & Forth & Fifth & Average \\
\hline 1 & $2.2529 \mathrm{e}+07$ & $2.0528 \mathrm{e}+07$ & $1.8986 \mathrm{e}+07$ & $1.4891 \mathrm{e}+07$ & $2.1349 \mathrm{e}+07$ & $1.96566 \mathrm{e}+07$ \\
2 & $1.3648 \mathrm{e}+07$ & $1.5863 \mathrm{e}+07$ & $1.5468 \mathrm{e}+07$ & $1.3876 \mathrm{e}+07$ & $1.7525 \mathrm{e}+07$ & $1.5276 \mathrm{e}+07$ \\
3 & $2.4251 \mathrm{e}+07$ & $1.6173 \mathrm{e}+07$ & $1.6144 \mathrm{e}+07$ & $1.7583 \mathrm{e}+07$ & $1.8199 \mathrm{e}+07$ & $1.847 \mathrm{e}+07$ \\
4 & $1.7896 \mathrm{e}+07$ & $1.9454 \mathrm{e}+07$ & $2.107 \mathrm{e}+07$ & $1.726 \mathrm{e}+07$ & $1.5458 \mathrm{e}+07$ & $1.82276 \mathrm{e}+07$ \\
5 & $2.2285 \mathrm{e}+07$ & $1.7558 \mathrm{e}+07$ & $1.6035 \mathrm{e}+07$ & $1.5751 \mathrm{e}+07$ & $2.0607 \mathrm{e}+07$ & $1.84472 \mathrm{e}+07$ \\
\hline
\end{tabular}

Normalizing the result data of different characteristic values In order to facilitate the analysis of the relationship between different eigenvalues. Fig.9 is the result of normalizating $X$ - ray diffraction stress value and MBNvalue, the residual value of MBN and $\mathrm{X}$ ray diffraction stress is consistent basically.

\section{Conclusion}

A variable magnetic excitation sensor device is developed which include detection of MMM, DC MFL, AC MFL and MBN. It can not only evaluate the defect state of the specimen, but also evaluate the residual stress state, which provides a direction for the further development of the magnetic nondestructive testing technology.

\section{Acknowledgment}

This work was financially supported by the National Natural Science Foundation of China (Grant No. 51275048). The authors are grateful to the referees for their helpful comments and suggestions.

\section{References}

[1] Lin JM.Development and New Progress on Electromagnetic Test Technology [J].ENGINEERING \& TEST,2011,51(1): 1-3

[2]Hu CX. Research on Key Technologies and Design ofIntegrated Eddy Current NondestructiveTesting System [D]. ChangSha: Graduate School of National University of Defense Technology, 2012.

[3]Wang ZD, Gu Y, Wang YS. A review of three magnetic NDT technologies. J Magn Magn Mater, 324(2012)382-388.

[4] Kong QX, Zhang ZH.Analysis of SET /RESET Circuits in AMR Sensors[J].Chinese Journal of Scientific Instrument, 2005, 26(8):118-119.

[5]Wang ZD,Gu Y, Wang YS. A review of three magnetic NDT technologies. J Magn Magn Mater, 324(2012)382-388.

[6]Jing T, Que PW.Noise Elimination of Magnetic Flux Leakage Inspecting Based on Wavelet Theory[J], Journal of Test and Measurement Technology, 2003, 17(4): 359-362.

[7] Zhang WM, Qiu ZC, Yuan JJ, Yu X. Discussion on Stress Quantitative Evaluation using Metal Magnetic Memory Method[J], Journal of Mechanical Engineering, 2015,51(8):9-13.

[8] Li W, Chen GM. Defect Visualization for Alternating Current Field Measurement Based on the Double U-shape Inducer Array, Journal of Mechanical Engineering, 2009,45(9):233-237.

[9]Muhammad A, Satish U. Advanced signal processing of magnetic flux leakage data obtained from seamless gas pipeline. NDT\&E International, 35(2002)449-45

[10] Yonka Ivanova,Todor Partalin.Comparative Measurements of the Stress State in a Rolled Carbon Steel Using Magnetic Barkhausen Noise and Ultrasonic Method[J].Russian Journal of Nondest ructive Testing,2012,48(2):137-146. 